\title{
Solvability and Asymptotic Behavior of Generalized Riccati Equations Arising in Indefinite Stochastic LQ Controls
}

\author{
Mustapha Ait Rami, Xi Chen, John B. Moore, Fellow, IEEE, and Xun Yu Zhou, Senior Member, IEEE
}

\begin{abstract}
The optimal control problem in a finite time horizon with an indefinite quadratic cost function for a linear system subject to multiplicative noise on both the state and control can be solved via a constrained matrix differential Riccati equation. In this paper, we provide general necessary and sufficient conditions for the solvability of this generalized differential Riccati equation. Furthermore, its asymptotic behavior is investigated along with its connection to the generalized algebraic Riccati equation associated with the linear quadratic control problem in infinite time horizon. Examples are presented to illustrate the results established.
\end{abstract}

Index Terms-Asymptotic analysis, generalized Riccati equation, indefinite stochastic linear quadratic (LQ) control, linear matrix inequality, solvability.

\section{INTRODUCTION}

$\mathbf{L}$ INEAR quadratic (LQ) control is one of the most fundamental and widely used tools in modern engineering. In recent years, the applications in fields such as mathematical finance require the study of stochastic LQ control models that are qualitatively different from traditional LQ models in that the control will affect not only the (deterministic) drift component of the system dynamics, but also the (stochastic) diffusion component. For instance, adjusting the position of certain assets held in a portfolio affects not only its return, but also its volatility. Furthermore, the direct cost for exercising the control could be zero or even negative, while finding the optimal control remains a meaningful problem, as the noise (diffusion) part of the state process will automatically deter any overzealous control actions.

To be specific, consider the following stochastic LQ problem:

Minimize

$$
\begin{aligned}
J(u(\cdot)):=\mathbf{E} \int_{t_{0}}^{T}[ & x(t)^{\prime} Q(t) x(t)+2 x(t)^{\prime} L(t) u(t) \\
& \left.+u(t)^{\prime} R(t) u(t)\right] d t+\mathbf{E}\left[x(T)^{\prime} M x(T)\right]
\end{aligned}
$$

subject to

$$
d x(t)=[A(t) x(t)+B(t) u(t)] d t
$$

Manuscript received October 1, 1999; revised June 7, 2000. Recommended by Associate Editor Q. Zhang. This work was supported by the RGC under Earmarked Grants CUHK 4125/97E and CUHK 4054/98E.

M. A. Rami, X. Chen, and X. Y. Zhou are with the Department of Systems Engineering and Engineering Management, Chinese University of Hong Kong, Shatin, Hong Kong (e-mail: aitm@se.cuhk.edu.hk; xchen@se.cuhk.edu.hk; xyzhou@se.cuhk.edu.hk).

J. B. Moore is with the Department of Systems Engineering, Australian National University, ACT 0200, Australia (e-mail: john.moore@anu.edu.au).

Publisher Item Identifier S 0018-9286(01)02565-X.

$$
\begin{aligned}
& +[C(t) x(t)+D(t) u(t)] d w(t) \\
x\left(t_{0}\right)= & x_{0} \in \mathbf{R}^{n} .
\end{aligned}
$$

Note that the above model has multiplicative white noises on both the state and control. In the special case when $C(\cdot) \equiv 0$, $D(\cdot) \equiv 0$, the system is a deterministic time-varying linear system, and it is well known that the LQ problem is meaningless if for almost every $t, R(t)$, the control weighting matrix in the cost, has at least one negative eigenvalue. However, recent studies [11], [12], [20] show that when $D(t) \neq 0$ the stochastic LQ problem could be still well posed even if $R$ is singular or indefinite. In fact, a singular or indefinite $R$ may naturally occur in a wide class of practical problems, ranging from portfolio selection [29], option pricing [19], to pollution control [11]. In addition, in some cases $R$ is identically zero as the control cost is only implicitly incurred by the underlying uncertainty; see [29], [19]. Another interesting case of the LQ problem is when $L$ is identically zero and $Q(t)$, the state weighting matrix in the cost, is negative while $R(t)$ is positive definite. This constitutes a generalization of the stochastic $H_{\infty}$ control problem [14], [25], [17].

The above indefinite stochastic LQ control problems lead to the following constrained nonlinear backward differential matrix equation:

$$
\left\{\begin{array}{l}
\dot{P}(t)+A(t)^{\prime} P(t)+P(t) A(t) \\
\quad+C(t)^{\prime} P(t) C(t)+Q(t) \\
\quad-\left[P(t) B(t)+C(t)^{\prime} P(t) D(t)+L(t)\right] \\
\quad \times\left[R(t)+D(t)^{\prime} P(t) D(t)\right]^{-1} \\
\quad \times\left[B(t)^{\prime} P(t)+D(t)^{\prime} P(t) C(t)+L(t)^{\prime}\right]=0 \\
P(T)=M, \\
R(t)+D(t)^{\prime} P(t) D(t)>0, \quad \text { a.e. } t \in[0, T] .
\end{array}\right.
$$

In this paper, we refer to this equation as a generalized differential Riccati equation (GDRE). It will play a central role in the treatment of the indefinite LQ problem. It is shown in [11] that,in the case when $L \equiv 0$, the solvability of the GDRE is sufficient for solving the LQ problem. Indeed, a unique optimal control, which has a linear state feedback structure, can be constructed explicitly based on the solution to the GDRE (1). Thus, solving the original indefinite LQ problem boils down to that of solving the GDRE. It should be noted that a Riccati equation was first derived by Bismut [7] for a stochastic LQ problem with 
control-dependent diffusions and random coefficients, however its solvability was proved, using a functional analysis approach, under the key assumption that the control cost $R$ is positive definite. A similar Riccati equation was later studied by Bensoussan [6], where the positive definiteness of $R$ was again imposed. A stochastic Riccati equation, formulated as a nonlinear backward stochastic differential equation (BSDE), was first introduced in [11] for indefinite stochastic LQ problems with random coefficients. While the solvability issue of this Riccati BSDE, as a very challenging problem, is yet to be resolved, some special cases, especially in the case of deterministic coefficients where the Riccati BSDE degenerates to the GDRE (1), have been settled. Specifically, in [11], a necessary and sufficient condition is given for the solvability of the GDRE for the case when $C \equiv 0$ and $L \equiv 0$ and, based on this condition, an algorithm of computing its solution is proposed. However, the algorithm assumes the availability of an initial solution which is in fact hard to locate. In the case when $C \neq 0$, some necessary condition for the LQ problem to be solvable is derived based on a decomposition approach in [12]. Nevertheless, solvability of the GDRE (1) in general remains, as cited in [11], as an outstanding open problem.

It is one of the objectives of this paper to tackle this open problem. First, we extend the result in [11] by showing that the existence of a unique optimal control to LQ problem is equivalent to the solvability of the GDRE (1). Next, we provide a necessary and sufficient condition for the solvability of the GDRE in terms of the feasibility of a certain linear matrix inequality (LMI). This LMI is different from the traditional one [8] for it is parameterized by the time $t$ and involves the derivative in $t$. The LMI condition derived can be interpreted as a generalization of the well-known real bounded lemma which plays a central role in the stochastic $H_{\infty}$ theory [14], [25], [17]. Indeed, the condition gives rise to qualitative information about the solvability of the GDRE. For example, it implies that the solvability is "convex" with respect to some problem parameters (e.g., the weighting matrices). It also leads to some comparison theorem for the solutions to the GDRE. More importantly, the condition suggests some numerical test of the solvability of the GDRE. One example is when all the coefficients are time-invariant, it suffices to check if there is any constant symmetric matrix satisfying the proposed LMI condition. This can be done efficiently via a semidefinite programming [24], [15].

Let us emphasize again that our results do not assume that the matrix function $R(\cdot)$ is coercive and/or continuous as commonly assumed in the literature. In this paper, $R(\cdot)$ is simply required to be an essentially bounded measurable function, as are the other parameters of the problem.

The next issue of interest is the asymptotic behavior of the solution to the GDRE (1) as the time horizon expands to be infinitely large, and its connection to the so-called generalized algebraic Riccati equation (GARE) explored extensively in [2]

$$
\left\{\begin{aligned}
A^{\prime} P & +P A+C^{\prime} P C+Q-\left(P B+C^{\prime} P D+L\right) \\
& \times\left(R+D^{\prime} P D\right)^{-1}\left(B^{\prime} P+D^{\prime} P C+L^{\prime}\right)=0 \\
R+ & D^{\prime} P D>0
\end{aligned}\right.
$$

where all the coefficient matrices are time-invariant. As wellknown GARE corresponds to the stochastic LQ problem in infinite time horizon [2]

$$
\begin{aligned}
& \text { Minimize } \\
& \begin{aligned}
J(u(\cdot)) & :=\mathbf{E} \int_{t_{0}}^{+\infty}\left[x(t)^{\prime} Q x(t)+2 x(t)^{\prime} L u(t)+u(t)^{\prime} R u(t)\right] d t \\
\text { subject to: } & \\
\qquad x(t) & =[A x(t)+B u(t)] d t+[C x(t)+D u(t)] d w(t) \\
x\left(t_{0}\right) & =x_{0} \in \mathbf{R}^{n}
\end{aligned}
\end{aligned}
$$

where an admissible control is such that the corresponding trajectory satisfies $\lim _{t \rightarrow+\infty} \mathbf{E}\left[x(t)^{\prime} x(t)\right]=0$. In other words, the input controls of the system are assumed to be (mean-square) stabilizing.

The asymptotic behavior of the differential Riccati equation in the classical deterministic LQ setting has been largely studied; see [9]. Although some open problems still remain [23], the asymptotic theory has been well established [26], [10]. However, the indefinite stochastic LQ case remains unexplored. In fact, the literature on the subject concerns only the definite case where there is no noise on the control. The first study can be traced back to [27], [28]. The paper [1] provides a convex optimization approach for solving the GARE (2) (with $D=0$, $L=0, Q \geq 0$ and $R>0$ ). A direct treatment of the infinite horizon stochastic LQ problem with multiplicative noise both on the state and the control $(C \neq 0, D \neq 0)$ with possibly singular $R \geq 0$ is also given in [1]. A recent paper [16] investigates the convergence properties of the "linearly perturbed" deterministic time-invariant Riccati equation introduced in [27] with positive $Q$ and $R$.

In this paper we carry out an asymptotic analysis for the indefinite stochastic LQ case. The results establish the link between finite and infinite time horizon LQ problems as well as that between the GDRE and GARE. We show that if the terminal condition of the GDRE (1) is a feasible point to certain LMI (which can be examined and solved by existing efficient numerical algorithm), then its corresponding solution exists, which converges monotonically to some solution of the corresponding GARE as the time horizon increases to infinity. The convergence to the maximal solution of the GARE is also investigated. We show that if there exists a terminal condition $P(T)=M$ (not necessarily positive and may be indefinite) such that the corresponding solution of the GDRE converges to the maximal solution of the GARE, then the convergence holds for any terminal condition "larger" than $M$. In particular, the existence and the convergence hold for any terminal condition larger than the maximal solution.

The rest of the paper is organized as follows. In Section II, we first formulate the indefinite stochastic LQ problem in finite time horizon and present some preliminaries. Then we show that the solvability of the GARE is necessary and sufficient for the existence and uniqueness of the optimal control. Section III is devoted to the solvability of the GDRE for three different cases. Some comparison theorems are also obtained. In Section IV, we 
consider the indefinite LQ problem in infinite time horizon and its associated GARE, carry out an asymptotic analysis for the GDRE, and establish its link to the GARE. In Section V, we give examples to illustrate the results obtained. Finally, Section VI concludes the paper.

\section{Problem Statement AND Preliminaries}

Notation: In this paper, the following notation is adopted:

\section{$\mathbf{R}^{n \times m}$}

$M^{\prime}$

$\operatorname{Tr}(M)$

$\operatorname{ker}(M)$

$|M|$

$\mathcal{S}^{n}$

$\mathcal{S}_{+}^{n}$

$\mathbf{E} x$

$L^{\infty}\left(a, b ; \mathbf{R}^{m \times p}\right)$ space of all $n \times m$ of real matrices; transpose of a matrix $M$; sum of diagonal elements of a square matrix $M$;

kernel of a matrix $M$;

$=\sqrt{\operatorname{Tr}\left(M M^{\prime}\right)}$

space of all $n \times n$ symmetric matrices; subspace of all nonnegative definite matrices of $\mathcal{S}^{n}$ expected value of a random variable $x$ set of all essentially bounded, Lebesgue measurable and $\mathbf{R}^{m \times p}$-valued functions on $[a, b]$.

Given a filtered probability space $\left(\Omega, \mathcal{F}, \mathcal{P} ; \mathcal{F}_{t}\right)$, where $t \in$ $[0, T]$, and a Hilbert space $X$ with the norm $\|\cdot\|_{X}$, define the Hilbert space

$$
\begin{aligned}
L_{\mathcal{F}}^{2}(0, T ; X)=\{\phi(\cdot) \mid & \phi(\cdot) \text { is an } \mathcal{F}_{t^{-}} \text {-adapted, } X \text {-valued } \\
& \text { measurable process on }[0, T], \\
& \text { and } \left.\mathbf{E} \int_{0}^{T}\|\phi(t, \omega)\|_{X}^{2} d t<+\infty\right\}
\end{aligned}
$$

with the norm

$$
\|\phi(\cdot)\|_{\mathcal{F}, 2}=\left(E \int_{0}^{T}\|\phi(t, \omega)\|_{X}^{2} d t\right)^{1 / 2}
$$

\section{A. Indefinite LQ Problem}

The GDRE (1) arises in the stochastic LQ control problem where the system dynamics is governed by the following linear stochastic differential equation

$$
\left\{\begin{aligned}
d x(t)= & {[A(t) x(t)+B(t) u(t)] d t } \\
& +[C(t) x(t)+D(t) u(t)] d w(t) \\
x\left(t_{0}\right)= & x_{0}
\end{aligned}\right.
$$

where $\left(t_{0}, x_{0}\right) \in[0, T) \times \mathbf{R}^{n}$ are, respectively, the initial time and initial state, and $w(\cdot)$ is a given one-dimensional (1-D) standard Brownian motion on $\left[t_{0}, T\right]$. Note that the results of the paper can be extended to the multidimensional Brownian motion case without essential difficulty. An admissible control $u(\cdot)$ is an $\mathbf{R}^{n_{u}}$-valued, $\mathcal{F}_{t}$-adapted measurable process. The set of all admissible controls is denoted by $U_{a d}$.
Associated with the above dynamics, we consider the quadratic cost function for $\left(t_{0}, x_{0}\right) \in[0, T) \times \mathbf{R}^{n}$ and $u(\cdot) \in U_{a d}$

$$
\begin{aligned}
J\left(t_{0},\right. & \left.x_{0} ; u(\cdot)\right) \\
= & \mathbf{E} \int_{t_{0}}^{T}\left[x(t)^{\prime} Q(t) x(t)+2 x(t)^{\prime} L(t) u(t)+u(t)^{\prime} R(t) u(t)\right] d t \\
& +\mathbf{E}\left[x(T)^{\prime} M x(T)\right] .
\end{aligned}
$$

The solution $x(\cdot)$ of the system (3) is called the response of the control $u(\cdot) \in U_{a d}$, and $(x(\cdot), u(\cdot))$ is called an admissible pair. The objective of the problem is to minimize the cost function $J\left(t_{0}, x_{0} ; u(\cdot)\right)$, for a given $\left(t_{0}, x_{0}\right) \in[0, T) \times \mathbf{R}^{n}$, over all $u(\cdot) \in U_{a d}$. The value function is defined as

$$
V\left(t_{0}, x_{0}\right)=\inf _{u(\cdot) \in U_{a d}} J\left(t_{0}, x_{0} ; u(\cdot)\right)
$$

An admissible pair $\left(x^{*}(\cdot), u^{*}(\cdot)\right)$ is called optimal if it achieves the infimum of $J\left(t_{0}, x_{0} ; u(\cdot)\right)$. The optimization problem (3) and (4) is called well posed if $V\left(t_{0}, x_{0}\right)>-\infty$, for all $\left(t_{0}, x_{0}\right) \in[0, T) \times \mathbf{R}^{n}$.

Throughout this paper, we make the following assumptions on the coefficients of the LQ problem (3) and (4)

$$
\left\{\begin{array}{l}
A(\cdot), C(\cdot) \in L^{\infty}\left(0, T ; \mathbf{R}^{n \times n}\right) \\
B(\cdot), D(\cdot), L(\cdot) \in L^{\infty}\left(0, T ; \mathbf{R}^{n \times n_{u}}\right) \\
Q(\cdot) \in L^{\infty}\left(0, T ; \mathcal{S}^{n}\right), R(\cdot) \in L^{\infty}\left(0, T ; \mathcal{S}^{n_{u}}\right) \\
M \in \mathcal{S}^{n}
\end{array}\right.
$$

Note that we have not assumed any definiteness of the above coefficients. We shall then categorize LQ problems into the following classes. An LQ problem is called

1) definite if $\left[\begin{array}{cc}Q(t) & L(t) \\ L(t)^{\prime} & R(t)\end{array}\right] \geq 0, R(t)>0$, a.e. $t \in[0, T]$, and $M \geq 0$

2) singular if $\left[\begin{array}{cc}Q(t) & L(t) \\ L(t)^{\prime} & R(t)\end{array}\right] \geq 0, R(t) \geq 0$ is singular, a.e. $t \in[0, T]$, and $M \geq 0$

3) indefinite if there is no restriction on the definiteness of $\left[\begin{array}{ll}Q(t) & L(t) \\ L(t)^{\prime} & R(t)\end{array}\right]$ and $M$.

It is easily verified that in both cases 1) and 2) the LQ problem is well posed. Moreover, the existence and uniqueness of an optimal control are guaranteed in the case 1), whereas it is not true for the case 2) where there may be no optimal control or infinitely many optimal controls. The general situation 3 ) is the most complicated one to analyze, whose solution is the main objective of this paper.

\section{B. LMI, GDRE and $L Q$}

In our analysis below, the following LMI will play a central role:

$$
\left[\begin{array}{c|c}
\dot{P}+A^{\prime} P+P A+C^{\prime} P C+Q & P B+C^{\prime} P D+L \\
\hline B^{\prime} P+D^{\prime} P C+L^{\prime} & R+D^{\prime} P D
\end{array}\right] \geq 0
$$


where $P(\cdot)$ is a continuously differentiable symmetric matrix function such that

$$
R(t)+D(t)^{\prime} P(t) D(t)>0, \quad \text { a.e. } t \in[0, T] \text {, and } P(T) \leq M \text {. }
$$

Let us start with the following lemma.

Lemma 2.1: Let $S(\cdot)$ be any continuously differentiable symmetric matrix function with respect to time and $(x(\cdot), u(\cdot))$ be an admissible pair of (3). Then, for any $t_{0}, t_{1} \in[0, T]$

$$
\begin{aligned}
\mathbf{E}[x & \left.\left(t_{1}\right)^{\prime} S\left(t_{1}\right) x\left(t_{1}\right)-x\left(t_{0}\right)^{\prime} S\left(t_{0}\right) x\left(t_{0}\right)\right] \\
= & \mathbf{E} \int_{t_{0}}^{t_{1}}\left[x^{\prime}\left(\dot{S}+A^{\prime} S+S A+C^{\prime} S C\right) x\right. \\
& \left.+2 u^{\prime}\left(B^{\prime} S+D^{\prime} S C\right) x+u^{\prime} D^{\prime} S D u\right](t) d t \\
= & \mathbf{E} \int_{t_{0}}^{t_{1}}\left[\begin{array}{l}
x \\
u
\end{array}\right]^{\prime}\left[\begin{array}{c|c}
\dot{S}+A^{\prime} S+S A+C^{\prime} S C & S B+C^{\prime} S D \\
\hline B^{\prime} S+D^{\prime} S C & D^{\prime} S D
\end{array}\right] \\
& \times\left[\begin{array}{l}
x \\
u
\end{array}\right](t) d t .
\end{aligned}
$$

Proof: By Ito's formula, we have (the argument $t$ is suppressed)

$$
\begin{aligned}
d\left(x^{\prime} S x\right)=[ & x^{\prime}\left(\dot{S}+A^{\prime} S+S A+C^{\prime} S C\right) x \\
& \left.+2 u^{\prime}\left(B^{\prime} S+D^{\prime} S C\right) x+u^{\prime} D^{\prime} S D u\right] d t \\
& +\left[x^{\prime}\left(C^{\prime} S+S C\right) x+u^{\prime}\left(D^{\prime} S+S D\right) u\right] d w(t) .
\end{aligned}
$$

Taking integrations and expectations we get (9).

Now, we establish a link between the well posedness of the LQ problem and the LMI condition (7) and (8).

Theorem 2.1: If there exists a symmetric matrix function $P(\cdot)$ satisfying (7) and (8), then the LQ problem (3) and (4) is well posed.

Proof: Using Lemma 2.1 and a simple manipulation we have, for any $u(\cdot) \in U_{a d}$

$$
\begin{aligned}
J\left(t_{0},\right. & \left.x_{0} ; u(\cdot)\right) \\
= & \mathbf{E} \int_{t_{0}}^{T}\left[\begin{array}{l}
x \\
u
\end{array}\right]^{\prime} \\
& \times\left[\begin{array}{l|c}
\dot{P}+A^{\prime} P+P A+C^{\prime} P C+Q & P B+C^{\prime} P D+L \\
\hline B^{\prime} P+D^{\prime} P C+L^{\prime} & R+D^{\prime} P D
\end{array}\right] \\
& \times\left[\begin{array}{l}
x \\
u
\end{array}\right](t) d t+x_{0}^{\prime} P x_{0}+\mathbf{E}\left[x(T)^{\prime}(M-P(T)) x(T)\right] \\
\geq & x_{0}^{\prime} P x_{0} .
\end{aligned}
$$

Hence, the LQ problem (3) and (4) is well posed.

Next, we show that a GDRE solution also ensures the well posedness of the LQ problem and, moreover, provides an optimal feedback control law.

Theorem 2.2: If the GDRE (1) admits a solution $P(\cdot)$, then the LQ problem (3) and (4) is well-posed. Moreover, there is a unique optimal control with the following feedback form:

$$
\begin{aligned}
u(t)= & -\left[R(t)+D(t)^{\prime} P(t) D(t)\right]^{-1} \\
& \times\left[B(t)^{\prime} P(t)+D(t)^{\prime} P(t) C(t)+L(t)^{\prime}\right] x(t) .
\end{aligned}
$$

Furthermore, the value function is given by

$$
V\left(t_{0}, x_{0}\right)=x_{0}^{\prime} P\left(t_{0}\right) x_{0}, \quad \forall\left(t_{0}, x_{0}\right) \in[0, T] \times \mathbf{R}^{n} .
$$

Proof: This result is proved in [11, Th. 3.2] for the case when $L \equiv 0$. But the proof is extended directly to the case $L \not \equiv 0$.

The preceding result shows that the solvability of the GDRE is sufficient for the existence and uniqueness of an optimal control to the LQ problem. Conversely, we are going to prove that the solvability of the GDRE is also necessary to the existence of a unique optimal control. To this end, we first make use of the main results in [3, Th. 3.1, Th. 5.2] which are summarized below.

Proposition 2.1 [3]: The following conditions are equivalent.

1) The LQ problem (3) and (4) has a unique optimal open-loop control for any initial condition $\left(t_{0}, x_{0}\right) \in[0, T] \times \mathbf{R}^{n}$.

2) There exists a solution $P(\cdot)$ to the following constrained differential equation (with the time argument $t$ suppressed)

$$
\left\{\begin{array}{l}
\dot{P}+P A+A^{\prime} P+C^{\prime} P C+Q-\left(P B+C^{\prime} P D+L\right) \\
\quad \times\left(R+D^{\prime} P D\right)^{\dagger}\left(B^{\prime} P+D^{\prime} P C+L^{\prime}\right)=0, \\
P(T)=M \\
\left(R+D^{\prime} P D\right)\left(R+D^{\prime} P D\right)^{\dagger}\left(B^{\prime} P+D^{\prime} P C+L^{\prime}\right) \\
\quad-\left(B^{\prime} P+D^{\prime} P C+L^{\prime}\right)=0, \\
R+D^{\prime} P D \geq 0, \quad \text { a.e. } t \in[0, T]
\end{array}\right.
$$

where $M^{\dagger}$ denotes the Moore-Penrose pseudo inverse of a matrix $M[21]$.

Moreover, the set of all the optimal controls with respect to the initial condition $\left(t_{0}, x_{0}\right) \in[0, T) \times \mathbf{R}^{n}$ is determined by the following [parameterized by $(Y, z)]$ :

$$
\begin{aligned}
u_{(Y, z)}(t)=- & \left\{\left[R(t)+D(t)^{\prime} P(t) D(t)\right]^{\dagger}\right. \\
& \times\left[B(t)^{\prime} P(t)+D(t)^{\prime} P(t) C(t)+L(t)^{\prime}\right] \\
& +Y(t)-\left[R(t)+D(t)^{\prime} P(t) D(t)\right]^{\dagger} \\
& \left.\times\left[R(t)+D(t)^{\prime} P(t) D(t)\right] Y(t)\right\} x(t) \\
+ & z(t)-\left[R(t)+D(t)^{\prime} P(t) D(t)\right]^{\dagger} \\
\times & {\left[R(t)+D(t)^{\prime} P(t) D(t)\right] z(t) }
\end{aligned}
$$

where $Y(\cdot) \in L_{\mathcal{F}}^{2}\left(0, T ; \mathbf{R}^{n_{u} \times n}\right)$ and $z(\cdot) \in L_{\mathcal{F}}^{2}\left(0, T ; \mathbf{R}^{n_{u}}\right)$.

Now, as an immediate consequence of Proposition 2.1, we have the following.

Theorem 2.3: The LQ problem (3) and (4) has a unique optimal control for any initial condition $\left(t_{0}, x_{0}\right) \in[0, T) \times \mathbf{R}^{n}$ if and only if the GDRE (1) admits a solution. Moreover, the optimal control is a linear state feedback given by (10).

Proof: The sufficiency part follows from Theorem 2.2. Now, we prove the solvability of the GDRE (1) assuming that the LQ problem has a unique optimal control. In view of Proposition 2.1 it suffices to prove that the matrix $\left[R(t)+D(t)^{\prime} P(t) D(t)\right]$ is nonsingular for a.e. $t \in[0, T]$. To this end, let $u^{*}(\cdot)$ be the unique optimal control with respect to 
the initial condition $\left(0, x_{0}\right)$, which by Proposition 2.1 is given by

$$
\begin{aligned}
u^{*}(t)=- & \left\{\left[R(t)+D(t)^{\prime} P(t) D(t)\right]^{\dagger}\right. \\
& \times\left[B(t)^{\prime} P(t)+D(t)^{\prime} P(t) C(t)+L(t)^{\prime}\right]+Y \\
& -\left[R(t)+D(t)^{\prime} P(t) D(t)\right]^{\dagger} \\
& \left.\times\left[R(t)+D(t)^{\prime} P(t) D(t)\right] Y\right\} x(t) \\
+ & z-\left[R(t)+D(t)^{\prime} P(t) D(t)\right]^{\dagger} \\
& \times\left[R(t)+D(t)^{\prime} P(t) D(t)\right] z
\end{aligned}
$$

where $Y \in \mathbf{R}^{n_{u} \times n}$ and $z \in \mathbf{R}^{n_{u}}$ are chosen to be arbitrary deterministic constants. By the uniqueness of $u^{*}(\cdot)$, it is necessary that

$$
\begin{aligned}
& I-\left[R(t)+D(t)^{\prime} P(t) D(t)\right]^{\dagger} \\
& \times\left[R(t)+D(t)^{\prime} P(t) D(t)\right]=0 \\
& \text { a.e. } t \in[0, T] .
\end{aligned}
$$

Noting that any symmetric matrix $M$ commutes with its pseudoinverse $M^{\dagger} M=M M^{\dagger}$ [21], we conclude from (14) that

$$
\begin{aligned}
& {\left[R(t)+D(t)^{\prime} P(t) D(t)\right]^{\dagger}\left[R(t)+D(t)^{\prime} P(t) D(t)\right]} \\
& \quad=\left[R(t)+D(t)^{\prime} P(t) D(t)\right]\left[R(t)+D(t)^{\prime} P(t) D(t)\right]^{\dagger} \\
& \quad=I, \quad \text { a.e. } t \in[0, T] .
\end{aligned}
$$

This implies that $\left[R(t)+D(t)^{\prime} P(t) D(t)\right]$ is nonsingular.

In general, a nonlinear differential equation may admit many solutions. However, when the GDRE has a solution it must be unique. In fact, the uniqueness of its solution seems to be inherent due to the connection between the GDRE and the LQ problem.

Corollary 2.1: If there exists a solution $P(\cdot)$ to the GDRE (1), then, it must be unique.

Proof: Let $P_{1}(\cdot)$ and $P_{2}(\cdot)$ be two solutions of (1) with $P_{1}(T)=P_{2}(T)=M$. Then, by Theorem 2.2, we have

$$
\begin{array}{r}
V\left(t_{0}, x_{0}\right)=x_{0}^{\prime} P_{1}\left(t_{0}\right) x_{0}=x_{0}^{\prime} P_{2}\left(t_{0}\right) x_{0} \\
\forall\left(t_{0}, x_{0}\right) \in[0, T] \times \mathbf{R}^{n} .
\end{array}
$$

Hence, $P_{1}(\cdot)=P_{2}(\cdot)$.

\section{EXISTENCE OF SOLUTION TO GDRE}

By Theorem 2.2, one only needs to solve the GDRE in order to solve the LQ problem. The aim of this section is to give conditions under which the GDRE has a solution. An important implication of our conditions is that they identify the set of all terminal points $P(T)=M$ such that the corresponding GDREs admit solutions. We will show that this set is convex and can be described by an LMI set. Another interesting aspect is that the solvability of the GDRE is "convex" with respect to the weighting matrices $L, Q$ and $R$.

In what follows, we solve the problem for three different cases. However, before that, we state the following lemma which will be used in the sequel.
Lemma 3.1 (Schur's Lemma [8]): Let matrices $X=X^{\prime}$, $Y=Y^{\prime}$ and $Z$ be given with appropriate dimensions. The following conditions are equivalent:
i) $X-Z Y^{-1} Z^{\prime} \geq 0, \quad Y>0$.
ii) $\left[\begin{array}{ll}X & Z \\ Z^{\prime} & Y\end{array}\right] \geq 0, \quad Y>0$

\section{A. Definite Case}

The solvability of the GDRE (1) in the definite case is a consequence of the solvability of a more general Riccati equation (with random coefficients) proved by Bismut [7]. However, we supply an independent proof here because it is much simpler in the present case of deterministic coefficients and, moreover, it is interesting to compare it with those in the singular and indefinite cases.

Theorem 3.1: Assume that $\left[\begin{array}{ll}Q(t) & L(t) \\ L(t)^{\prime} & R(t)\end{array}\right] \geq 0, R(t)>0$, a.e. $t \in[0, T]$, and $M \geq 0$. Then, the GDRE (1) has a solution $P(\cdot)$ on $[0, T]$ with $P(t) \geq 0, \forall t \in[0, T]$.

Proof: By the classical ordinary differential equation theory, the GDRE (1) has a local solution $P(\cdot)$ on some maximal interval $\left(t_{m}, T\right] \subset[0, T]$. Since $R(t)>0$, the inequality constraint in (1) is satisfied automatically at any $t$ when $P(t)$ exists. Hence in order to prove that the existence is actually global on $[0, T]$, it suffices to show that there is no escape time, or $P(\cdot)$ is uniformly bounded on $\left(t_{m}, T\right]$. To this end, we are going to show that there exists a positive scalar $\beta>0$ independent of $t_{m}$ such that

$$
0 \leq P(t) \leq \beta I, \quad \forall t \in\left(t_{m}, T\right]
$$

First, to see that $P(\cdot) \geq 0$ let $x_{0} \in \mathbf{R}^{n}$ be an arbitrary initial state of the system (3) starting at a time $t \in\left(t_{m}, T\right]$. Then, Theorem 2.2 implies

$$
\begin{gathered}
x_{0}^{\prime} P(t) x_{0}=\min _{u(\cdot) \in U_{a d}} \mathbf{E}\left\{\int_{t}^{T}\left[\begin{array}{l}
x \\
u
\end{array}\right]^{\prime}\left[\begin{array}{ll}
Q & L \\
L^{\prime} & R
\end{array}\right]\left[\begin{array}{l}
x \\
u
\end{array}\right](s) d s\right. \\
\left.+x(T)^{\prime} M x(T)\right\} \geq 0 .
\end{gathered}
$$

Next, let $x(\cdot)$ be a solution to the system (3) corresponding to the initial $\left(t, x_{0}\right)$ and the admissible control $u(\cdot) \equiv 0$. Then, Theorem 2.2 implies

$$
x_{0} P(t) x_{0} \leq \mathbf{E}\left[\int_{t}^{T} x^{\prime}(s) Q(s) x(s) d s+x(T)^{\prime} M x(T)\right]
$$

From the above inequality, and the fact that $x(\cdot)$ satisfies a homogeneous linear equation, it follows that there exists a scalar $\beta>0$ such that $x_{0}^{\prime} P(t) x_{0} \leq \beta x_{0}^{\prime} x_{0}$. The proof is completed. $\diamond$

\section{B. Singular Case}

In the singular case, the GDRE (1) no longer admits a solution automatically, as in the definite case. We will give conditions that ensure the existence. 
Theorem 3.2: Assume that $\left[\begin{array}{ll}Q(t) & L(t) \\ L(t)^{\prime} & R(t)\end{array}\right] \geq 0$, a.e. $t \in[0, T]$, and $M \geq 0$. Moreover, assume that either $L(t) \equiv 0$ and $Q(t)>$ $0, \forall t \in[0, T]$, or $M>0$. Then, the GDRE (1) has a solution $P(\cdot)$ on $[0, T]$ with $P(t) \geq 0, \forall t \in[0, T]$, if and only if

$$
\operatorname{ker} R(t) \cap \operatorname{ker} D(t)=\{0\}, \quad \text { a.e. } t \in[0, T) \text {. }
$$

Proof: The "only if" part is straightforward. Now, let us prove the "if" part and suppose that (15) holds. Let the GDRE (1) have a local solution on some maximal interval $\left(t_{m}, T\right] \subset$ $[0, T]$. Define $P\left(t_{m}\right)$ as $\lim _{t \rightarrow t_{m}}+P\left(t_{m}\right)$. In fact, this limit is well defined and can be determined via

$$
\begin{aligned}
& x\left(t_{m}\right)^{\prime} P\left(t_{m}\right) x\left(t_{m}\right) \\
&=\mathbf{E}\left\{\int _ { t _ { m } } ^ { T } \left[x_{*}(s)^{\prime} Q(s) x_{*}(s)+2 x_{*}(s)^{\prime} L(s) u_{*}(s)\right.\right. \\
&\left.\left.\quad+u_{*}(s)^{\prime} R(s) u_{*}(s)\right] d s+x_{*}(T)^{\prime} M x_{*}(T)\right\}
\end{aligned}
$$

where $\left(x_{*}(\cdot), u_{*}(\cdot)\right)$ is an optimal pair corresponding to an arbitrary initial state $x\left(t_{m}\right)$ at the initial time $t_{m}$. Next, we show that $R\left(t_{m}\right)+D\left(t_{m}\right)^{\prime} P\left(t_{m}\right) D\left(t_{m}\right)>0$ or, as $P\left(t_{m}\right) \geq 0$, that $R\left(t_{m}\right)+D\left(t_{m}\right)^{\prime} P\left(t_{m}\right) D\left(t_{m}\right)$ is nonsingular. Suppose that there is $0 \neq x_{0} \in \mathbf{R}^{n}$ so that $\left[R\left(t_{m}\right)+D\left(t_{m}\right)^{\prime} P\left(t_{m}\right) D\left(t_{m}\right)\right] x_{0}=0$. Then

$$
x_{0}^{\prime}\left[R\left(t_{m}\right)+D\left(t_{m}\right)^{\prime} P\left(t_{m}\right) D\left(t_{m}\right)\right] x_{0}=0
$$

implying $x_{0}^{\prime} R\left(t_{m}\right) x_{0}=0$ and $x_{0}^{\prime} D\left(t_{m}\right)^{\prime} P\left(t_{m}\right) D\left(t_{m}\right) x_{0}=0$. It follows that $x_{0} \in \operatorname{ker} R\left(t_{m}\right)$ (noting that $R\left(t_{m}\right) \geq 0$ ) and hence by (15), $D\left(t_{m}\right) x_{0} \neq 0$ (otherwise, $x_{0}=0$ leading to a contradiction). Consider the system (3) starting at the time $t_{m}$ with the initial state $D\left(t_{m}\right) x_{0}$. Then, Theorem 2.2 yields

$$
\begin{aligned}
& 0 \equiv x_{0}^{\prime} D\left(t_{m}\right)^{\prime} P\left(t_{m}\right) D\left(t_{m}\right) x_{0} \\
&=\mathbf{E}\left\{\int _ { t _ { m } } ^ { T } \left[x_{*}(s)^{\prime} Q(s) x_{*}(s)+2 x_{*}(s)^{\prime} L(s) u_{*}(s)\right.\right. \\
&\left.\left.\quad+u_{*}(s)^{\prime} R(s) u_{*}(s)\right] d s+x_{*}(T)^{\prime} M x_{*}(T)\right\}
\end{aligned}
$$

where $\left(x_{*}(\cdot), u_{*}(\cdot)\right)$ is the unique optimal pair solution to the LQ problem on $\left(t_{m}, T\right]$, with $u_{*}(t)=$ $-\left(R+D^{\prime} P D\right)^{-1}\left(B^{\prime} P+D^{\prime} P C+L^{\prime}\right)(t) x_{*}(t)$. Since the initial state $x_{*}\left(t_{m}\right)=D\left(t_{m}\right) x_{0} \neq 0$, we claim that

$$
x_{*}(t) \neq 0, \quad P-a . s ., \quad \forall t \in\left[t_{m}, T\right] .
$$

Indeed, by Ito's formula, it is easy to see that $\mathbf{E}\left[x_{*}(t) x_{*}(t)^{\prime}\right]$ satisfies a linear equation on $\left[t_{m}, T\right]$. Hence, it will be identically zero should it be zero at any time instant, contracting to the fact that $x_{*}\left(t_{m}\right) \neq 0$. This proves (17).

To proceed, we consider two cases according to the assumption of the theorem.

Case 1) $L(t) \equiv 0, Q(t)>0$. The equality (16) implies that the integrand on its right-hand side must be zero almost surely on $\left[t_{m}, T\right]$. Since $Q(t)>0$, it must hold that $x_{*}(t)=0, P-a . s$., a.e. $t \in\left[t_{m}, T\right]$. This contradicts (17).

Case 2) $M>0$. Again (16) leads to $x_{*}(T)^{\prime} M x_{*}(T)=0$, $P-$ a.s.. Hence, $x_{*}(T)=0$, which is a contraction.

Finally, the same argument as in the proof of Theorem 3.1 can be used to show that $P(\cdot)$ is uniformly bounded on $\left(t_{m}, T\right]$, completing the proof.

Remark 3.1: The condition (15) is equivalent to the inequality $R(t)+D(t)^{\prime} D(t)>0$, which can be checked by calculating the smallest eigenvalue $\lambda_{\min }\left(R(t)+D(t)^{\prime} D(t)\right)$. If $R(t) \equiv 0$, then the condition (15) reduces to that $D(\cdot)$ has a full column rank almost everywhere or, equivalently, that $D(t)^{\prime} D(t)>0$, a.e. $t \in[0, T)$. On the other hand, if $D(t) \equiv 0$ (meaning that the control does not enter into the diffusion term), then (15) is equivalent to the standard positive definite condition $R(t)>0$, a.e. $t \in[0, T)$.

\section{Indefinite Case}

In this section, we consider the general indefinite case. We will show how we can reduce this case to the definite one.

Consider the following convex set $\mathcal{P}_{M}$ of $\mathcal{S}^{n}$-valued functions, parameterized by the terminal condition $M$ as shown in (18) at the bottom of the page.

We have the following result.

Theorem 3.3: The GDRE (1) [with the terminal condition $P(T)=M]$ has a solution if and only if $\mathcal{P}_{M} \neq \emptyset$.

Proof: If the Riccati equation (1) has a solution $P$, then, $P(T)=M$ and, by Lemma 3.1, we have

$$
\left[\begin{array}{c|c}
\dot{P}+A^{\prime} P+P A+C^{\prime} P C+Q & P B+C^{\prime} P D+L \\
\hline B^{\prime} P+D^{\prime} P C+L^{\prime} & R+D^{\prime} P D
\end{array}\right] \geq 0 .
$$

Namely, $P \in \mathcal{P}_{M} \neq \emptyset$.

Conversely, suppose $\mathcal{P}_{M} \neq \emptyset$. Then, take any $\tilde{P} \in \mathcal{P}_{M}$ and define

$$
\left\{\begin{array}{l}
Q(\tilde{P})=\dot{\tilde{P}}+A^{\prime} \tilde{P}+\tilde{P} A+C^{\prime} \tilde{P} C+Q \\
R(\tilde{P})=R+D^{\prime} \tilde{P} D \\
L(\tilde{P})=\tilde{P} B+C^{\prime} \tilde{P} D+L
\end{array}\right.
$$

$$
\mathcal{P}_{M} \triangleq\left\{\begin{array}{l|l}
\tilde{P}(\cdot):[0, T] \rightarrow \mathcal{S}^{n}, \quad \tilde{P}(T) \leq M, \\
{\left[\begin{array}{c|c}
\tilde{\tilde{P}}+A^{\prime} \tilde{P}+\tilde{P} A+C^{\prime} \tilde{P} C+Q & \tilde{P} B+C^{\prime} \tilde{P} D+L \\
\hline B^{\prime} \tilde{P}+D^{\prime} \tilde{P} C+L^{\prime} & R+D^{\prime} \tilde{P} D
\end{array}\right] \geq 0} \\
R+D^{\prime} \tilde{P} D>0, & \text { a.e. } t \in[0, T]
\end{array}\right\} .
$$


Thus, $R(\tilde{P})>0$. Moreover, Lemma 3.1 yields

$$
Q(\tilde{P})-L(\tilde{P}) R(\tilde{P})^{-1} L(\tilde{P})^{\prime} \geq 0
$$

Now, consider a new Riccati equation

$$
\left\{\begin{array}{l}
\dot{Z}_{\tilde{P}}+A^{\prime} Z_{\tilde{P}}+Z_{\tilde{P}} A+C^{\prime} Z_{\tilde{P}} C+Q(\tilde{P}) \\
-\left[Z_{\tilde{P}} B+C^{\prime} Z_{\tilde{P}} D+L(\tilde{P})\right]\left[R(\tilde{P})+D^{\prime} Z_{\tilde{P}} D\right]^{-1} \\
\quad\left[B^{\prime} Z_{\tilde{P}}+D^{\prime} Z_{\tilde{P}} C+L(\tilde{P})^{\prime}\right]=0 \\
R(\tilde{P})+D^{\prime} Z_{\tilde{P}} D>0, \\
Z_{\tilde{P}}(T)=M-\tilde{P}(T) \geq 0 .
\end{array}\right.
$$

By Theorem 3.1, there exists a unique positive solution $Z_{\tilde{P}}(\cdot)$ to (19). Define $P(\cdot)=Z_{\tilde{P}}(\cdot)+\tilde{P}(\cdot)$. Then clearly $R+D^{\prime} P D>$ 0 and $P(T)=M$. Using equation (19) and some manipulation, we can easily verify that $P(\cdot)$ is a solution to the GDRE (1). $\diamond$

The following two corollaries concern the solvability of the GDRE (1) with the data $M$ and $L, Q$, and $R$ regarded as parameters.

Corollary 3.1: The set of all terminal conditions $M$ such that the GDRE (1) admits a solution is unbounded from above and is convex.

Proof: The unboundedness follows from Theorem 3.3 and the fact that $\mathcal{P}_{M} \subset \mathcal{P}_{\tilde{M}}$ for any $\tilde{M} \geq M$. The convexity is straightforward by Theorem 3.3 .

Corollary 3.2: The set of all weighting matrices $L, Q$ and $R$ such that the GDRE (1) admits a solution is unbounded from above and is convex.

Proof: This is straightforward by Theorem 3.3.

Corollary 3.3: If $P$ is the solution to the GDRE (1) with a terminal condition $P(T)=M$, then $P(\cdot) \geq \tilde{P}(\cdot) \quad \forall \tilde{P}(\cdot) \in$ $\mathcal{P}_{M}$.

Proof: Let $\tilde{P} \in \mathcal{P}_{M}$ be arbitrary and $Z_{\tilde{P}} \geq 0$ be the solution to (19). Then, $P=Z_{\tilde{P}}+\tilde{P}$ solves the GDRE (1) by the proof of Theorem 3.3, and it is the unique solution by Corollary 2.1. It follows that $P-\tilde{P}=Z_{\tilde{P}} \geq 0$ and the proof is complete.

Corollary 3.4 (Comparison Theorem): If $P_{1}(\cdot)$ and $P_{2}(\cdot)$ are two solutions of the GDRE (1) with $P_{1}(T) \leq P_{2}(T)$, then

$$
P_{1}(t) \leq P_{2}(t) \quad \forall t \in[0, T]
$$

Proof: This is implied by the fact that $P_{1}(\cdot) \in \mathcal{P}_{P_{2}(T)}$ along with Corollary 3.3.

Before concluding this section, we compare our results with those obtained in a preceding paper [11]. In [11], it is proved that when $R$ is indefinite but $C=0, L=0, Q \geq 0$ and $M \geq 0$, the GDRE admits a solution if and only if the following condition holds: there exists $K=K^{\prime}>0$ such that the standard deterministic Riccati equation

$$
\left\{\begin{array}{l}
\dot{P}+A^{\prime} P+P A-P B K^{-1} B^{\prime} P+Q=0 \\
P(T)=M
\end{array}\right.
$$

has a solution $P$ satisfying

$$
R+D^{\prime} P D \geq K
$$

Now, we are going to show that in the special setting of [11] our general condition $\mathcal{P}_{M} \neq \emptyset$ reduces to the condition of [11] mentioned above. In fact, our condition even leads to a relaxation of (20) with the "=" in the first equality of (20) replaced by " $\geq$." To this end, first let $P$ satisfy (20) and (21). Then, by Lemma 3.1, we have

$$
\left[\begin{array}{cc}
\dot{P}+A^{\prime} P+P A+Q & P B \\
B^{\prime} P & K
\end{array}\right] \geq 0, \quad P(T)=M .
$$

Since $R+D^{\prime} P D \geq K>0$, it follows that

$$
\begin{aligned}
& {\left[\begin{array}{cc}
\dot{P}+A^{\prime} P+P A+Q & P B \\
B^{\prime} P & R+D^{\prime} P D
\end{array}\right] \geq 0} \\
& R+D^{\prime} P D>0, \quad P(T)=M \text {. }
\end{aligned}
$$

Hence, $\mathcal{P}_{M} \neq \emptyset$. Conversely, assume that $\tilde{P} \in \mathcal{P}_{M} \neq \emptyset$. Define $K=R+D^{\prime} \tilde{P} D>0$, and

$$
\tilde{Q}=\dot{\tilde{P}}+A^{\prime} \tilde{P}+\tilde{P} A-\tilde{P} B K^{-1} B^{\prime} \tilde{P}+Q \geq 0 .
$$

The above simply means that $\tilde{P}$ solves the following standard Riccati equation:

$$
\dot{\tilde{P}}+A^{\prime} \tilde{P}+\tilde{P} A-\tilde{P} B K^{-1} B^{\prime} \tilde{P}+Q-\tilde{Q}=0
$$

Now, let $P$ be the solution to

$$
\dot{P}+A^{\prime} P+P A-P B K^{-1} B^{\prime} P+Q=0, \quad P(T)=M .
$$

Since $Q \geq Q-\tilde{Q}$ and $M \geq \tilde{P}(T)$, by the well-known comparison theorem for standard Riccati equation (see, e.g., [22]) we have $P(\cdot) \geq \tilde{P}(\cdot)$. This, in particular, implies that $R+D^{\prime} P D \geq$ $R+D^{\prime} \tilde{P} \bar{D}=K$.

\section{ASYMPTOTIC BEHAVIOR OF GDRE}

In this section, we carry out asymptotic analysis to the GDRE (1) and investigate its relation to the GARE which is associated with the stochastic LQ problem in infinite time horizon. The asymptotic properties of the GDRE reveal valuable information on the evolution of a finite time horizon LQ problem as the horizon increases. More importantly, the asymptotic analysis will establish links between the indefinite stochastic LQ problems in a finite time horizon and the infinite time horizon. The main result in this section is the convergence of the solution of the GDRE (1) to the so-called maximal solution of the corresponding GARE.

Since GARE is to be involved, we need to assume that all the matrices $A, B, C, D, L, Q, R$ in (3) and (4) are time invariant. From now on, we denote by $P(\cdot, T)$ the solution of (1) with the parameterized terminal time $T$. Note that since all the coefficients of (1) are time invariant, $P(t, T)$ may exist for $t \in$ $(-\infty,+\infty)$. We are going to study the $\operatorname{limit}_{t \rightarrow-\infty} P(t, T)$ which by time-invariance is equal to $\lim _{(T-t) \rightarrow+\infty} P(0, T-t)$ or simply $\lim _{T \rightarrow+\infty} P(0, T)$. 


\section{A. Mean-Square Stabilizability}

First, we define the notion of mean-square stabilizability, which is connected to the stochastic LQ problem for the infinite time horizon case [2].

Definition 4.1: The system (3) is said to be mean-square stabilizable if there exists a control law of feedback form

$$
u(t)=K x(t), \quad K \in \mathbf{R}^{n_{u} \times n}
$$

with $K$ being a constant matrix, such that for every initial $\left(t_{0}, x_{0}\right)$, the closed-loop system

$$
\left\{\begin{array}{l}
d x(t)=(A+B K) x(t) d t+(C+D K) x(t) d w(t) \\
x\left(t_{0}\right)=x_{0}, \quad x_{0} \in \mathbf{R}^{n}
\end{array}\right.
$$

satisfies

$$
\lim _{t \rightarrow+\infty} \mathbf{E}\left[x(t)^{\prime} x(t)\right]=0 .
$$

In this case, the control law given by (23) is called a mean-square stabilizing control.

In the infinite horizon case, the cost function may in general be unbounded from above in the absence of the mean-square stabilizability, in which case the optimal control problem becomes ill-posed. Hence the analysis of the asymptotic properties of the GDRE will be carried out under the following natural condition of mean-square stabilizability of the system. We will show that this assumption guarantees the boundedness of the solutions to the GDRE. When there is no noise on the system (i.e., $C \equiv 0, D \equiv 0$ ), this assumption reduces to the well-known deterministic stabilizability condition of the pair $(A, B)$.

Assumption 4.1: The system (3) is mean-square stabilizable.

Remark 4.1: The following condition is equivalent to Assumption 4.1 [2]: There exists a matrix $K$ and a symmetric matrix $X$ such that

$$
\begin{aligned}
& (A+B K) X+X(A+B K)^{\prime}+(C+D K) X(C+D K)^{\prime}<0 \\
& \quad X>0 .
\end{aligned}
$$

Moreover, by the change of variable $Y=K X$ and using Schur's lemma one obtains an equivalent LMI condition: there exists a matrix $Y$ and a symmetric matrix $X$ such that

$$
\left[\begin{array}{cc}
A X+X A^{\prime}+B Y+Y^{\prime} B^{\prime} & C X+D Y \\
X C^{\prime}+Y^{\prime} D^{\prime} & -X
\end{array}\right]<0 .
$$

This also provides a mean-square stabilizing feedback control $u(t)=Y X^{-1} x(t)$.

To prove the boundedness and the asymptotic convergence of the solutions to the GDRE we will make use of the following lemma which at the same time gives another equivalent condition to Assumption 4.1.

Lemma 4.1: The following properties are equivalent:

1) system (3) is mean-square stabilizable;

2) there is a constant matrix $K$ such that the solution of (24) satisfies $\mathbf{E}\left[\int_{t_{0}}^{+\infty} x(t)^{\prime} x(t) d t\right]<+\infty$, for any $\left(t_{0}, x_{0}\right)$.

Proof: That 2$) \Rightarrow 1$ ) is obvious. To show the opposite implication, suppose that the system (3) is mean-square stabilizable by a feedback matrix $K$. Denote $X(t)=\mathbf{E}\left[x(t) x(t)^{\prime}\right]$ where $x(\cdot)$ satisfies the system equation (24). By Ito's formula, $X(\cdot)$ satisfies the following linear differential equation:

$$
\left\{\begin{array}{c}
\dot{X}=(A+B K) X+X(A+B K)^{\prime} \\
+(C+D K) X(C+D K)^{\prime} \\
X\left(t_{0}\right)=x_{0} x_{0}^{\prime}
\end{array}\right.
$$

The mean-square stabilizability condition $\lim _{t \rightarrow+\infty} \mathbf{E}\left[x(t)^{\prime} x(t)\right] \quad=\quad 0 \quad$ implies $\lim _{t \rightarrow+\infty} X(t)=\lim _{t \rightarrow+\infty} \mathbf{E}\left[x(t) x(t)^{\prime}\right]=0$. Hence, $X(\cdot)$ is an asymptotically stable solution to a linear, time-invariant deterministic system (27). It follows then there exist positive constants $\alpha$ and $\beta$ such that

$$
|X(t)|=\left|\mathbf{E}\left[x(t) x(t)^{\prime}\right]\right|<\beta e^{-\alpha t} .
$$

Hence, the desired result follows.

\section{B. Solvability of GDRE and GARE}

In this section, we characterize the solvability of GDRE and GARE in terms of certain LMI conditions. Define the following nonlinear operator $\mathcal{R}$ from $\mathcal{S}^{n} \times \mathbf{R}^{n \times n_{u}} \times \mathcal{S}^{n} \times \mathcal{S}^{n_{u}}$ to $\mathcal{S}^{n}$

$$
\begin{aligned}
\mathcal{R}(P, L, Q, R) & \\
\triangleq & A^{\prime} P+P A+C^{\prime} P C+Q-\left(P B+C^{\prime} P D+L\right) \\
& \times\left(R+D^{\prime} P D\right)^{-1}\left(B^{\prime} P+D^{\prime} P C+L^{\prime}\right) .
\end{aligned}
$$

Recall that the GARE (2) can be rewritten as

$$
\mathcal{R}(P, L, Q, R)=0 \quad \text { and } \quad R+D^{\prime} P D>0 .
$$

Let us define the following subset of $\mathcal{S}^{n}$ as shown in (30) at the bottom of the page.

The following result shows that any element of the set $\mathcal{P}_{L, Q, R}$ leads to solutions to the GDRE (1) as well as the GARE (29).

Theorem 4.1: Assume that $\mathcal{P}_{L, Q, R} \neq \emptyset$. Then, for any terminal condition $P(T)=\tilde{P}$ with $\stackrel{P}{P} \in \mathcal{P}_{L, Q}, R$, the solution $P(\cdot) \equiv P(\cdot, T)$ to the GDRE (1) exists and is bounded on $(-\infty, T]$. Moreover, $P(t)$ is monotonically nondecreasing as

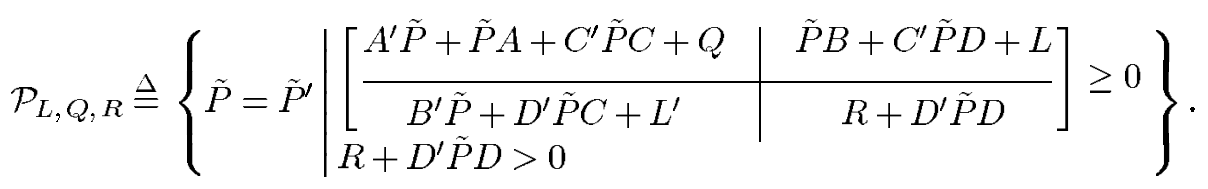


time $t$ decreases, and converges to a solution $P$ to the GARE (29) as $t \rightarrow-\infty$.

Proof: Fix $\tilde{P} \in \mathcal{P}_{L, Q, R}$. Denote by $Z_{\tilde{P}}(\cdot, T)$ the solution of the differential Riccati equation (19) with $Z_{\tilde{P}}(T, T)=0$. Denote

$$
\left\{\begin{array}{l}
Q(\tilde{P})=A^{\prime} \tilde{P}+\tilde{P} A+C^{\prime} \tilde{P} C+Q \\
R(\tilde{P})=D^{\prime} \tilde{P} D+R \\
L(\tilde{P})=\tilde{P} B+C^{\prime} \tilde{P} D+L
\end{array}\right.
$$

Now, Let $x(t)=x_{0}$ be arbitrary and consider the following cost function for the system (3):

$$
J_{\tilde{P}}\left(t, x_{0} ; u(\cdot)\right)=\mathbf{E} \int_{t}^{T}\left[\begin{array}{l}
x \\
u
\end{array}\right]^{\prime}\left[\begin{array}{ll}
Q(\tilde{P}) & L(\tilde{P}) \\
L(\tilde{P})^{\prime} & R(\tilde{P})
\end{array}\right]\left[\begin{array}{l}
x \\
u
\end{array}\right](s) d s .
$$

Applying Theorem 2.2, we have

$$
x_{0}^{\prime} Z_{\tilde{P}}(t, T) x_{0}=\inf _{u(\cdot) \in U_{a d}} J_{\tilde{P}}(t, x(t) ; u(\cdot)) .
$$

Since the matrix $\left[\begin{array}{cc}Q(\tilde{P}) & L(\tilde{\Gamma}) \\ L(\tilde{P})^{\prime} & R(\tilde{P})\end{array}\right]$ is nonnegative, for any $t_{1} \leq t_{2}$ we have

$$
Z_{\tilde{P}}\left(t_{2}, T\right) \leq Z_{\tilde{P}}\left(t_{1}, T\right)
$$

namely, $Z_{\tilde{P}}(t, T)$ monotonically nondecreases as $t$ decreases.

Observe that by time invariance of the system, $Z_{\tilde{P}}(t, T)$ depends only on $(T-t)$. To be precise, we have the following equality

$$
Z_{\tilde{P}}(t, T)=Z_{\tilde{P}}(0, T-t), \quad \forall t<T .
$$

Let $u(t)=K x(t)$ be an arbitrary stabilizing control. Then by Assumption 4.1 and Lemma 4.1, we have

$$
\begin{aligned}
& x_{0}^{\prime} Z_{\tilde{P}}(t, T) x_{0} \\
& \quad \equiv x_{0}^{\prime} Z_{\tilde{P}}(0, T-t) x_{0} \\
& \leq \mathbf{E} \int_{0}^{T-t}\left[\begin{array}{c}
x \\
K x
\end{array}\right]^{\prime}\left[\begin{array}{ll}
Q(\tilde{P}) & L(\tilde{P}) \\
L(\tilde{P})^{\prime} & R(\tilde{P})
\end{array}\right]\left[\begin{array}{c}
x \\
K x
\end{array}\right](s) d s \\
& \leq \lambda_{\max }\left(\left[\begin{array}{c}
I \\
K
\end{array}\right]^{\prime}\left[\begin{array}{ll}
Q(\tilde{P}) & L(\tilde{P}) \\
L(\tilde{P})^{\prime} & R(\tilde{P})
\end{array}\right]\left[\begin{array}{c}
I \\
K
\end{array}\right]\right) \\
& \\
& \quad \int_{0}^{+\infty} \cdot \mathbf{E}\left[x(s)^{\prime} x(s)\right] d s \\
& <+\infty
\end{aligned}
$$

where $\lambda_{\max }(G)$ denotes the maximum eigenvalue of a matrix $G$. Thus, $Z_{\tilde{\Gamma}}(t, T)$ is bounded and nondecreasing as $t \rightarrow-\infty$. As a result, the following limit exists

$$
\bar{Z}_{\tilde{P}}=\lim _{t \rightarrow-\infty} Z_{\tilde{P}}(t, T)=\lim _{T-t \rightarrow+\infty} Z_{\tilde{P}}(0, T-t)
$$

which is constant and independent of the terminal time $T$. Letting $t \rightarrow-\infty$ in (19), we conclude that $\bar{Z}_{\tilde{P}}$ satisfies the following GARE:

$$
\left\{\begin{array}{l}
\mathcal{R}\left(\bar{Z}_{\tilde{P}}, L(\tilde{P}), Q(\tilde{P}), R(\tilde{P})\right)=0 \\
R(\tilde{P})+D^{\prime} \bar{Z}_{\tilde{P}} D>0
\end{array}\right.
$$

Now, define $P(t, T)=Z_{\tilde{P}}(t, T)+\tilde{P}$. Clearly, $P(t, T)$ is monotonically nondecreasing as $t$ decreases, and there exists a $\bar{P}$ such that

$$
\bar{P}=\lim _{t \rightarrow-\infty} P(t, T)=\bar{Z}_{\tilde{P}}+\tilde{P} .
$$

Combining (33) and (34) it follows from a simple calculation that $\bar{P}$ is the solution of (29).

The following shows that starting from a terminal state in the set $\mathcal{P}_{L, Q, R}$ the trajectory of the solution to the GDRE (1) stays in this set at any time backward.

Corollary 4.1: Assume that $\mathcal{P}_{L, Q, R} \neq \emptyset$. Then for any terminal condition $P(T)=\tilde{P}$ with $\tilde{P} \in \mathcal{P}_{L, Q, R}$, the solution $P(\cdot)$ to the GDRE (1) satisfies

$$
P(t) \in \mathcal{P}_{L, Q, R} \quad \forall t<T .
$$

Proof: By Theorem 4.1, $P(t)$ is monotonically nondecreasing as $t$ decreases. Hence, $\dot{P}(t) \leq 0, \forall t<T$. The result then follows from Lemma 3.1.

The following result, which establishes the equivalence between the nonemptiness of the set $\mathcal{P}_{L, Q, R}$ and the solvability of the GARE (29), has been proved in [2, Th. 5.6] (for the case $L=0$ ) by optimization techniques and a regularization argument. Here, we give another simple proof via the results just obtained.

Corollary 4.2: There exists a solution to the GARE (29) if and only if $\mathcal{P}_{L, Q, R} \neq \emptyset$.

Proof: If there exists a solution $P$ to the GARE (29), then clearly $P \in \mathcal{P}_{L, Q, R}$ in view of Lemma 3.1. Conversely, if $\mathcal{P}_{L, Q, R} \neq \emptyset$, then Theorem 4.1 applies to yield the solvability of (29).

\section{Maximal Solution of GARE}

Definition 4.2: A solution to the GARE (29) is called a maximal solution, denoted by $P_{\max }$, if

$$
P_{\max } \geq \tilde{P} \quad \forall \tilde{P} \in \mathcal{P}_{L, Q, R}
$$

Definition 4.3: A solution $P$ to the GARE (29) is called a stabilizing solution if the feedback control

$$
u(t)=-\left(R+D^{\prime} P D\right)^{-1}\left(B^{\prime} P+D^{\prime} P C+L^{\prime}\right) x(t)
$$

is mean-square stabilizing for the system (3).

By using the completion of square technique, we show that a stabilizing solution to the GARE, if there is any, must be the maximal solution.

Theorem 4.2: The stabilizing solution to the GARE (if there is any) is unique and coincides with the maximal solution.

Proof: Let $P$ be a stabilizing solution. It then suffices to show that $P_{\max } \leq P$. To this end, let $u(t)=K x(t) \equiv-(R+$ $\left.D^{\prime} P D\right)^{-1}\left(B^{\prime} P+D^{\prime} P C+L^{\prime}\right) x(t)$, which is a stabilizing feedback control by the assumption, and $x(\cdot)$ be the corresponding state trajectory starting from $x\left(t_{0}\right)=x_{0}$. By stability we have 
$\lim _{t \rightarrow+\infty} \mathbf{E}\left[x(t)^{\prime} x(t)\right]=0$. Applying Lemma 2.1 with $S(t) \equiv$ $P$, we have

$$
\begin{aligned}
& \mathbf{E} \int_{t_{0}}^{+\infty}\left[x(t)^{\prime} Q x(t)+2 x(t)^{\prime} L u(t)+u(t)^{\prime} R u(t)\right] d t \\
& =x_{0}^{\prime} P x_{0}-\lim _{t \rightarrow+\infty} \mathbf{E}\left[x(t)^{\prime} P x(t)\right] \\
& \quad+\mathbf{E} \int_{t_{0}}^{+\infty}[u(t)-K x(t)]^{\prime}\left(R+D^{\prime} P D\right)[u(t)-K x(t)] d t \\
& =x_{0}^{\prime} P x_{0} .
\end{aligned}
$$

Now, applying Lemma 2.1 with $S(t) \equiv P_{\max }$ and using the same manipulation as above, we get

$$
\begin{aligned}
x_{0}^{\prime} P x_{0}= & \mathbf{E} \int_{t_{0}}^{+\infty}\left[x(t)^{\prime} Q x(t)+2 x(t)^{\prime} L u(t)+u(t)^{\prime} R u(t)\right] d t \\
= & x_{0}^{\prime} P_{\max } x_{0}+\mathbf{E} \int_{t_{0}}^{+\infty}\left[u(t)-K_{\max } x(t)\right]^{\prime} \\
& \cdot\left(R+D^{\prime} P_{\max } D\right)\left[u(t)-K_{\max } x(t)\right] d t \\
\geq & x_{0}^{\prime} P_{\max } x_{0}
\end{aligned}
$$

where $K_{\max }=-\left(R+D^{\prime} P_{\max } D\right)^{-1}\left(B^{\prime} P_{\max }+D^{\prime} P_{\max } C+\right.$ $\left.L^{\prime}\right)$. The above inequality shows that $P \geq P_{\max }$ as the initial condition $x_{0}$ is arbitrary.

Before going further in the analysis of the asymptotic convergence of the GDRE to the maximal solution to the GARE some important remarks are in order.

Remark 4.2: The maximal solution or the stabilizing solution (if it exists) can be determined numerically by the following semidefinite programming [2, Th. 5.6]:

\section{$\max \operatorname{Tr}(P)$}

subject to

$$
\left\{\begin{array}{l|c}
{\left[\begin{array}{c|c}
A^{\prime} P+P A+C^{\prime} P C+Q & P B+C^{\prime} P D+L \\
\hline B^{\prime} P+D^{\prime} P C+L^{\prime} & R+D^{\prime} P D
\end{array}\right] \geq 0,} \\
R+D^{\prime} P D>0 .
\end{array}\right.
$$

Remark 4.3: The importance of the maximal solution to the GARE is that it corresponds to the optimal cost value for the infinite time horizon LQ problem. In other words, while there may be many solutions to the GARE, it is only the maximal solution that is interesting to us in view of the LQ problem. Moreover, the maximal solution gives bounds to the optimal cost value for the finite horizon case. Indeed, by virtue of the comparison theorem (Corollary 3.4), the solution to the GDRE with a terminal condition $P(T) \leq P_{\max }$ has an upper bound $P_{\max }$ whereas that with a terminal condition $P(T) \geq P_{\max }$ has a lower bound $P_{\max }$.

The following theorem has been proved in [2, Ths. 5.3, 5.4] for the case $L=0$, by using a duality analysis for an associated semidefinite programming problem. For the general case $L \neq 0$ while the proof there appear to be extendible, we supply here a different (but simpler) proof based on the asymptotic analysis of the corresponding GDRE.

Theorem 4.3: Assume that the set $\mathcal{P}_{L_{2} Q, R}$ has a nonempty interior, i.e., there exists $\tilde{P}$ such that $\mathcal{R}(\tilde{P}, L, Q, R)>0$ and
$D^{\prime} \tilde{P} D+R>0$. Then, the GARE (29) admits a stabilizing solution.

Proof: Let $\tilde{P}$ such that $\mathcal{R}(\tilde{P}, L, Q, R)>0, D^{\prime} \tilde{P} D+$ $R>0$. Consider $Z_{\tilde{P}}(\cdot)$, which is the solution to (19) with the terminal condition $Z_{\tilde{P}}(T)=0$ (which exists by Theorem 3.1). As in the proof of Theorem 4.1, we see that $Z_{\tilde{P}}(t)$ nondecreases and asymptotically converges (as $t$ decreases to $-\infty$ ) to some constant symmetric matrix $\bar{Z}_{\tilde{P}}$ that solves the following GARE:

$\mathcal{R}\left(\bar{Z}_{\tilde{P}}, L(\tilde{P}), Q(\tilde{P}), R(\tilde{P})\right)=0, \quad R(\tilde{P})+D^{\prime} \bar{Z}_{\tilde{P}} D>0$

where $Q(\tilde{P}), R(\tilde{P})$ and $L(\tilde{P})$ are defined as in (31).

Now, since $P=\bar{Z}_{\tilde{P}}+\tilde{P}$ solves the GARE (29) and

$$
\begin{aligned}
& {\left[R(\tilde{P})+D^{\prime} \bar{Z}_{\tilde{P}} D\right]^{-1}\left[B^{\prime} \bar{Z}_{\tilde{P}}+D^{\prime} \bar{Z}_{\tilde{P}} C+L(\tilde{P})^{\prime}\right]} \\
& \quad=\left(R+D^{\prime} P D\right)^{-1}\left(B^{\prime} P+D^{\prime} P C+L^{\prime}\right)
\end{aligned}
$$

it suffices to prove that the feedback control law

$$
u(t)=-\left[R(\tilde{P})+D^{\prime} \bar{Z}_{\tilde{P}} D\right]^{-1}\left[B^{\prime} \bar{Z}_{\tilde{P}}+D^{\prime} \bar{Z}_{\tilde{P}} C+L(\tilde{P})^{\prime}\right] x(t)
$$

is mean-square stabilizing. To this end, first observe that $\bar{Z}_{\tilde{P}}$ is positive definite

$$
\begin{aligned}
x_{0}^{\prime} \bar{Z}_{\tilde{P}} x_{0} & \geq x_{0}^{\prime} Z_{\tilde{P}}(s) x_{0} \\
& =\mathbf{E} \int_{s}^{T}\left[\begin{array}{l}
x \\
u
\end{array}\right]^{\prime}\left[\begin{array}{ll}
Q(\tilde{P}) & L(\tilde{P}) \\
L(\tilde{P})^{\prime} & R(\tilde{P})
\end{array}\right]\left[\begin{array}{l}
x \\
u
\end{array}\right](t) d t>0 .
\end{aligned}
$$

Define $K_{\tilde{P}}=-\left[R(\tilde{P})+D^{\prime} \bar{Z}_{\tilde{P}} D\right]^{-1}\left[B^{\prime} \bar{Z}_{\tilde{P}}+D^{\prime} \bar{Z}_{\tilde{P}} C+\right.$ $\left.L(\tilde{P})^{\prime}\right]$. Then, $K_{\tilde{P}}$ satisfies

$$
\begin{aligned}
(A+ & \left.B K_{\tilde{P}}\right)^{\prime} \bar{Z}_{\tilde{P}}+\bar{Z}_{\tilde{P}}\left(A+B K_{\tilde{P}}\right) \\
& +\left(C+D K_{\tilde{P}}\right)^{\prime} Z_{\tilde{P}}\left(C+D K_{\tilde{P}}\right) \\
= & -Q(\tilde{P})-K_{\tilde{P}}^{\prime} L(\tilde{P})^{\prime}-L(\tilde{P}) K_{\tilde{P}}-K_{\tilde{P}}^{\prime} R(\tilde{P}) K_{\tilde{P}} \\
= & -\left[\begin{array}{c}
I \\
K_{\tilde{P}}
\end{array}\right]^{\prime}\left[\begin{array}{ll}
Q(\tilde{P}) & L(\tilde{P}) \\
L(\tilde{P})^{\prime} & R(\tilde{P})
\end{array}\right]\left[\begin{array}{c}
I \\
K_{\tilde{P}}
\end{array}\right] .
\end{aligned}
$$

Since $\left[\begin{array}{c}I \\ K_{\tilde{P}}\end{array}\right]$ has a full column rank and $\left[\begin{array}{cc}Q(\tilde{P}) & L(\tilde{P}) \\ L(\tilde{P})^{\prime} & R(\tilde{P})\end{array}\right]$ is positive definite we have

$$
\begin{aligned}
& \left(A+B K_{\tilde{P}}\right)^{\prime} \bar{Z}_{\tilde{P}}+\bar{Z}_{\tilde{P}}\left(A+B K_{\tilde{P}}\right) \\
& \quad+\left(C+D K_{\tilde{P}}\right)^{\prime} \bar{Z}_{\tilde{P}}\left(C+D K_{\tilde{P}}\right)<0
\end{aligned}
$$

which by Remark 4.1 implies that $u(t)=K_{\tilde{P}} x(t)$ is meansquare stabilizing and the proof is complete.

The following result establishes the existence of the maximal solution to the GARE (29).

Theorem 4.4: Let $Q=Q^{\prime}, R=R^{\prime}$ and $L$ be given such that $\mathcal{P}_{L, Q, R} \neq \emptyset$, then the GARE (29) has a maximal solution.

Proof: This can be proved by using Theorem 4.3 and a regularization argument as in the proof of [2, Th. 5.6].

\section{Asymptotic Analysis of GDRE}

Theorem 4.1 gives a convergence of the solution of the GDRE to $a$ solution of the GARE. This, however, is not sufficient since, as mentioned earlier, only the maximal solution of the GARE is important in terms of ultimately solving the LQ problem. In this 
subsection we present the main results of this section, namely, the convergence of the solution of the GDRE to the maximal solution of the GARE.

First, we need a few lemmas.

Lemma 4.2: Assume that

$$
\left[\begin{array}{ll}
Q_{1} & L_{1} \\
L_{1}^{\prime} & R_{1}
\end{array}\right] \leq\left[\begin{array}{ll}
Q_{2} & L_{2} \\
L_{2}^{\prime} & R_{2}
\end{array}\right]
$$

and $\mathcal{P}_{L_{1}, Q_{1}, R_{1}} \neq \emptyset$. Then, there exist $P_{1}$ and $P_{2}$ satisfying

$$
\begin{cases}\mathcal{R}\left(P_{1}, L_{1}, Q_{1}, R_{1}\right)=0, & R_{1}+D^{\prime} P_{1} D>0 \\ \mathcal{R}\left(P_{2}, L_{2}, Q_{2}, R_{2}\right)=0, & R_{2}+D^{\prime} P_{2} D>0 \\ P_{1} \leq P_{2} . & \end{cases}
$$

Moreover, $P_{1}$ and $P_{2}$ are the maximal solutions of their respective GAREs.

Proof: This can be proved by using Theorems 4.3 and 4.2 , and an argument similar to that in the proof of [2, Lemma 5.1]. $\diamond$

The following lemma was first proposed in [16], but only for the case when there is no multiplicative noise on the control (i.e., $D=0$ ) and the weighting matrices are such that $L=0, Q \geq 0$ and $R>0$ (namely, a standard definite LQ case). Here, we present the result in a general setting.

Lemma 4.3: Let $Q=Q^{\prime}, R=R^{\prime}$ and $L$ be given. Assume that the solution $P(\cdot)$ of the GDRE (1) exists on $(-\infty, T]$ and for some $\tilde{t} \leq T$ there is a neighborhood $\delta(\tilde{t})$ of $\tilde{t}$ such that $P(t)$ is monotonically nondecreasing (resp. nonincreasing) as $t$ decreases in $\delta(\tilde{t})$. Then, $P(t)$ is monotonically nondecreasing (resp. nonincreasing) as $t$ decreases in $(-\infty, \tilde{t}]$.

Proof: In view of Corollary 3.4 and the time-invariance, the same argument in the proof of [16, Lemma 5.3-(ii)] applies.

Lemma 4.4: Let $M$ be an arbitrary symmetric matrix such that $R+D^{\prime} M D>0$. Then there exists an $\alpha>0$ such that the following GARE

$$
\mathcal{R}(P, L, Q+\alpha I, R)=0, \quad R+D^{\prime} P D>0
$$

admits a symmetric solution $P_{\alpha}$ with $P_{\alpha} \geq M$.

Proof: It can be easily seen that given $M=M^{\prime}$ there exists a sufficiently large $\alpha>0$ such that $\mathcal{R}(M, L, Q+\alpha I, R) \geq$ 0 . This, along with the assumption that $R+D^{\prime} M D>0$, leads to the existence of the maximal solution, $P_{\alpha}$, to the GARE

$$
\mathcal{R}\left(P_{\alpha}, L, Q+\alpha I, R\right)=0, \quad D^{\prime} P_{\alpha} D+R>0
$$

by virtue of Theorem 4.4. Finally, $P_{\alpha} \geq M$ follows from the maximality of $P_{\alpha}$.

Define

$$
\mathcal{M}_{L, Q, R} \triangleq\left\{M=M^{\prime} \mid \exists P \in \mathcal{P}_{L, Q, R} \text { such that } M \geq P\right\} .
$$

Theorem 4.5: Assume that $\mathcal{P}_{L, Q, R} \neq \emptyset$ and let $M \in \mathcal{M}_{L, Q, R}$ be given. Then there exists $\tilde{M} \geq M$ such that the GDRE (1) with the terminal condition $P(T)=\tilde{M}$ admits a solution $P(\cdot)$ on $(-\infty, T]$. Moreover, $P(t)$ is monotonically nonincreasing as $t$ decreases, and converges to the maximal solution to the GARE (29) as $t \rightarrow-\infty$.
Proof: By Lemma 4.4 there exists $\alpha>0$ and $P_{\alpha}$ such that

$$
\mathcal{R}\left(P_{\alpha}, L, Q+\alpha I, R\right)=0, \quad D^{\prime} P_{\alpha} D+R>0
$$

and $P_{\alpha} \geq M$. Let $\tilde{M}=P_{\alpha}$ and consider the GDRE

$$
\dot{P}+\mathcal{R}(P, L, Q, R)=0, \quad R+D^{\prime} P D>0
$$

with the terminal condition $P(T)=\tilde{M}$. This equation must have a solution $P(\cdot)$ on $(-\infty, T]$ in view of Theorem 4.1 and Corollary 3.1. Notice that at time $T$ the above GDRE specifies as $\dot{P}(T)-\alpha I=0$. Hence $\dot{P}(T)>0$ in a neighborhood of $T$. It follows from Lemma 4.3 that $P(t)$ is monotonically nonincreasing as $t$ decreases in $(-\infty, T]$. Now, let $P_{\max }$ be the maximal solution to the GARE (29). Then, Lemma 4.2 implies that $P(T) \geq P_{\max }$, which in turn yields that $P(t) \geq P_{\max }$ by Corollary 3.4. Since $P(t)$ is nonincreasing and bounded below as $t$ decreases, the limit $\lim _{t \rightarrow-\infty} P(t)=\bar{P}$ exists and $\bar{P}$ is easily seen to be a solution to the GARE (29). Moreover, $\bar{P} \geq P_{\max }$. By the maximality of $P_{\max }$ we conclude that $\bar{P}=P_{\max }$. The proof is complete.

Finally, the following theorem stipulates that Theorem 4.5 still holds for any terminal condition "larger" than the maximal solution of the GARE. More generally, if there exists some terminal condition $P(T)=\tilde{M}$ such that the corresponding solution to the GDRE converges to the maximal solution of the GARE, then the convergence still holds for any other terminal condition larger than $\tilde{M}$.

Theorem 4.6: Let $P_{\max }$ be the maximal solution to the GARE (29). Then, the GDRE (1) with a terminal condition $P(T) \geq P_{\max }$ admits a solution $P(\cdot)$, and $P(t)$ converges to $P_{\max }$ as $t \rightarrow-\infty$. More generally, if a solution of the GDRE (1) with a terminal condition $P(T)=M$ converges to $P_{\max }$ as the time goes to $-\infty$, then any solution of the GDRE (1) with a terminal condition $P(T) \geq M$ also converges to $P_{\max }$.

Proof: Let $P(\cdot)$ be a solution to the GDRE (1) with $P(T)=M \geq P_{\max }$, which exists by Theorem 4.1 and Corollary 3.1. Theorem 4.5 yields that there exists $\tilde{M} \geq M \geq P_{\max }$ such that $\tilde{P}(t)$ converges to $P_{\max }$ as $t \rightarrow-\infty$, where $\tilde{P}(\cdot)$ solves the GDRE (1) with the terminal condition $\tilde{P}(T)=\tilde{M}$. Now, Corollary 3.4 implies that

$$
\tilde{P}(t) \geq P(t) \geq P_{\max }, \quad \forall t<T .
$$

Hence, the first part of the theorem follows from the fact that $\lim _{t \rightarrow-\infty} \tilde{P}(t)=P_{\max }$. The second part of the theorem can be proved similarly.

\section{ILLUSTRATIVE EXAMPLES}

In this section, we present two examples to illustrate the results obtained. Consider the following 1-D LQ problem with constant coefficients $a, b, c, d, m, q, r$

$$
\begin{aligned}
& \text { Minimize } \\
& J=\mathbf{E}\left\{\int_{t_{0}}^{T}\left[q x(t)^{2}+r u(t)^{2}\right] d t+m x(T)^{2}\right\}
\end{aligned}
$$

subject to 


$$
\left\{\begin{array}{l}
d x(t)=[a x(t)+b u(t)] d t+[c x(t)+d u(t)] d W(t) \\
x\left(t_{0}\right)=x_{0}
\end{array}\right.
$$

Before going further, let us see how Assumption 4.1 translates into. By Remark 4.1 the mean-square stabilizability is equivalent to the following condition: There exists a scalar $k \in \mathbf{R}$, such that $f(k)=d^{2} k^{2}+2(b+c d) k+\left(c^{2}+2 a\right)<0$ or that the maximum of the function $f(k)$ is negative. This leads to the following inequality:

$$
2 a d^{2}-2 b c d-b^{2}<0 .
$$

Example 6.1: Consider the case where the coefficients of the system satisfy $b+c d=0, d \neq 0,2 a+c^{2}<0$. It is easy to verify that in this case (42) holds.

Assume that the weighting constants in the cost function are chosen from the following set:

$$
\left\{(m, q, r) \mid r\left(2 a+c^{2}\right)-q d^{2}<0,-\frac{r}{d^{2}} \leq m\right\} .
$$

The GDRE (1) reads

$\dot{p}(t)=-\left(2 a+c^{2}\right) p(t)-q, \quad p(T)=m$, and $r+d^{2} p(t)>0$.

With the data $(m, q, r)$ satisfying (43), it is easy to see that there is a $\hat{p}>-\left(r / d^{2}\right)$ such that $\hat{p} \in \mathcal{P}_{L, Q, R} \subset \mathcal{P}_{M}$. Hence Theorem 3.3 yields that the above equation admits a solution. Indeed this solution can be explicitly obtained as

$$
p(t)=\left(m+\frac{q}{2 a+c^{2}}\right) e^{-\left(2 a+c^{2}\right)(t-T)}-\frac{q}{2 a+c^{2}},
$$

where it can be directly verified that $r+d^{2} p(t)>0, \forall t \leq T$. Now, $\lim _{t \rightarrow-\infty} p(t)=-\left(q /\left(2 a+c^{2}\right)\right)$, which is exactly the maximal solution of the corresponding GARE

$$
\left(2 a+c^{2}\right) p+q=0, \quad r+d^{2} p>0 .
$$

Example 6.2: Now, assume that the system satisfies (42) and

$$
\left(d^{2} q+2 a r+c^{2} r\right)^{2}-4 q r\left(2 a d^{2}-2 b c d-b^{2}\right) \geq 0 .
$$

The above condition means that the equality part of the GARE

$$
\left(2 a d^{2}-b^{2}-2 b c d\right) p^{2}+\left(d^{2} q+2 a r+c^{2} r\right) p+q r=0
$$

admits two real solutions, say $p_{1}$ and $p_{2}$. If the control weight $r$ is such that $r+d^{2} \min \left\{p_{1}, p_{2}\right\}>0$, then $\max \left\{p_{1}, p_{2}\right\}$ is the maximal solution to the GARE and the set $\mathcal{P}_{L, Q}, R$ is exactly the interval $\left[\min \left\{p_{1}, p_{2}\right\}, \max \left\{p_{1}, p_{2}\right\}\right]$. Moreover, for any terminal condition $p(T)=m>\min \left\{p_{1}, p_{2}\right\}$, Theorem 4.1 implies that the corresponding solution to GDRE exists and nondecreasingly converges to a solution of the GARE, which in the present case must be $\max \left\{p_{1}, p_{2}\right\}$ for there are only two solutions to the GARE.

Let us look at a specific case with the following parameter values: $a=-(1 / 2), b=-3, c=1, d=2, q=8, r=-5$, whereas $m$ not yet fixed. The corresponding GDRE and GARE are

$$
\begin{gathered}
\dot{p}(t)=\frac{p(t)^{2}}{4 p(t)-5}-8, \quad p(T)=m, \quad 4 p(t)-5>0, \\
p^{2}-32 p+40=0, \quad 4 p-5>0
\end{gathered}
$$

respectively. The solutions to the GARE are $p_{1}=16-6 \sqrt{6}$ and $p_{2}=16+6 \sqrt{6}$. The maximal solution to the GARE is therefore $p_{\max }=16+6 \sqrt{6}$. The GDRE can be rewritten as

$$
\left\{\begin{array}{l}
\dot{p}(t)=\frac{(p(t)-16+6 \sqrt{6})(p(t)-16-6 \sqrt{6})}{4 p(t)-5} \\
p(T)=m, \quad 4 p(t)-5 \geq 0 .
\end{array}\right.
$$

By examining the sign of the function $f(v)=((v-16+$ $6 \sqrt{6})(v-16-6 \sqrt{6}) / 4 v-5)$ it can be easily seen that $p(t)$ nondecreasingly converges to $16+6 \sqrt{6}$ when $16-6 \sqrt{6}<m<$ $16+6 \sqrt{6}$, whereas $p(t)$ decreasingly converges to $16+6 \sqrt{6}$ when $m \geq 16+6 \sqrt{6}$.

\section{CONCLUDING REMARKS}

In this paper, we have characterized the solvability of a generalized differential Riccati equation, which is crucial to solving the indefinite stochastic LQ control problems that were first put forth in [11], in terms of a parameterized (the time $t$ being the parameter) LMI involving the derivative in $t$. The condition we obtained reduces to the one given in [11] in the special setting of [11]. Moreover, it gives rise to qualitative information on the solvability with respect to the problem data.

From the computational point of view, the generalized differential Riccati equation is much harder than the generalized algebraic Riccati equation for which a systematic numerical approach was proposed in [2]. Solving the parametrized LMI proposed in this paper appears to be a big challenge to researchers in both control and mathematical programming areas.

\section{REFERENCES}

[1] M. Ait Rami and L. El Ghaoui, "LMI optimization for stochastic Riccati equation,” IEEE Trans. Automat. Contr., vol. 41, pp. 1666-1671, Nov. 1996.

[2] M. Ait Rami and X. Y. Zhou, "Linear matrix inequalities, Riccati equations, and indefinite stochastic linear quadratic control," IEEE Trans. Automat. Contr., vol. 45, pp. 1131-1143, June 2000.

[3] M. Ait Rami, J. B. Moore, and X. Y. Zhou, "Indefinite stochastic linear quadratic control and generalized differential Riccati equation,", Preprint.

[4] A. Albert, "Conditions for positive and nonnegative definiteness in terms of pseudo-inverse," SIAM J. Appl. Math., vol. 17, pp. 434-440, 1969.

[5] M. Athans, "Special issues on linear-quadratic-Gaussian problem," IEEE Trans. Automat. Contr., vol. AC-16, pp. 527-869, Dec. 1971.

[6] A. Bensoussan, "Lecture on stochastic control-Part I," Lecture Notes in Math., vol. 972, pp. 1-39, 1983.

[7] J.-M. Bismut, "Linear quadratic optimal stochastic control with random coefficients," SIAM J. Contr. Optim., vol. 14, pp. 419-444, 1976.

[8] S. Boyd, L. El Ghaoui, E. Feron, and V. Balakrishnan, Linear Matrix Inequality in Systems and Control Theory. Philadelphia, PA: SIAM, 1994.

[9] S. Bittanti, A. J. Laub, and J. C. Willems, The Riccati Equation. New York: Springer-Verlag, 1991.

[10] F. M. Callier and J. L. Willems, "Criterion for the convergence of the solution of the Riccati Differential equation," IEEE Trans. Automat. Contr., vol. AC-26, pp. 1232-1242, Dec. 1981. 
[11] S. Chen, X. Li, and X. Y. Zhou, "Stochastic linear quadratic regulators with indefinite control weight costs," SIAM J. Control Optim., vol. 36, pp. $1685-1702,1998$

[12] S. Chen and X. Y. Zhou, "Stochastic linear quadratic regulators with indefinite control weight costs-II," SIAM J. Control Optim., vol. 39, pp. 1065-1081, 2000 .

[13] M. H. A. Davis, Linear Estimation and Stochastic Control. London, U.K.: Chapman and Hall, 1977.

[14] A. El Bouhtouri and A. J. Prichard, "Stability radii of linear systems with respect to stochastic perturbations," Syst. Control Lett., vol. 21, pp. 29-33, 1992.

[15] L. El Ghaoui, R. Nikoukhah, and F. Delebecque. (1995) LMITOOL: A front-end for LMI optimization in matlab. [Online]Available via anonymous ftp to . Available: ftp.ensta.fr, under /pub/elghaoui/lmitool

[16] M. D. Fragoso, O. L. V. Costa, and C. E. de Souza, "A new approach to linearly perturbed Riccati equations arising in stochastic control," Appl. Math. Optim., vol. 37, pp. 99-126, 1998.

[17] D. Hinrichsen and A. J. Prichard, "Stochastic $H^{\infty}$," SIAM J. Control Optim., vol. 36, pp. 1504-1538, 1998.

[18] R. E. Kalman, "Contribution to the theory of optimal control," Bol. Soc. Mat. Mex., vol. 5, pp. 102-119, 1960.

[19] M. Kohlmann and X. Y. Zhou, "Relationship between backward stochastic differential equations and stochastic controls: A linear-quadratic approach," SIAM J. Control Optim., vol. 38, pp. 1392-1407, 2000.

[20] A. E. B. Lim and X. Y. Zhou, "Optimal stochastic LQR control with integral quadratic constraints and indefinite control weights," IEEE Trans. Automat. Contr., vol. 44, pp. 1359-1369, July 1999.

[21] R. Penrose, "A generalized inverse of matrices," in Proc. Cambridge Philos. Soc., vol. 52, 1955, pp. 17-19.

[22] A. Ran and R. Vreugdenhil, "Existence and comparison theorems for algebraic Riccati equations for continuous and discrete time systems," Linear Algebra Appl., vol. 99, pp. 63-83, 1988.

[23] H. L. Trentelman et al., "When does the algebraic Riccati equation have a negative semi-definite solution," in Open Problems in Mathematical Systems and Control Control Theory, V. D. Blondel et al., Eds. New York: Springer-Verlag, 1999, pp. 229-237.

[24] L. Vandenerghe and S. Boyd, "Semidefinite programming," SIAM Review, 1996.

[25] V. A. Ugrinovskii, "Robust $H_{\infty}$ control in the presence of stochastic uncertainty," Int. J. Control, vol. 71, pp. 219-237, 1998

[26] J. C. Willems, "Least squares stationary control and the algebraic Riccati equation," IEEE Trans. Automat. Contr., vol. AC-16, pp. 621-634, Dec. 1971.

[27] W. M. Wonham, "On a matrix Riccati equation of stochastic control," SIAM J. Control, vol. 6, pp. 312-326, 1968.

[28] —, "Random differential equations in control theory," in In Probabilistic Methods in Applied Mathematics, A. T. B. Reid, Ed. New York: Academic, 1970, vol. 2, pp. 131-212.

[29] X. Y. Zhou and D. Li, "Continuous-time mean-variance portfoli selection: A stochastic LQ framework," Appl. Math. Optim., vol. 42, pp. $19-33,2000$

Mustapha Ait Rami was born in Marrakech, Morocco, in 1964. He received a first Maitrise (B.Sc.) degree in applied mathematics from Université Hassan II, Casablanca, Morocco, and a second Maitrise in pure mathematics from Université Cadi Ayad, Marrakech, Morocco. He received the DEA (M.S.) degree in nonlinear analysis-optimization and the Ph.D. degree in applied mathematics from the CEREMADE's Department, Université Paris-IX Dauphine, in 1997.

From 1997 to 1998, he worked on an applied project for EDF Company, France, at the Department de Mathematiques Appliquées, ENSTA, Paris, France. From 1998 to 1999 , he did postdoctoral research at the Chinese University of Hong Kong. His research interests are in the areas of robust control theory and optimal stochastic control. His research activities include also rank minimization problems and its connections with automatic control and semidefinite programs.

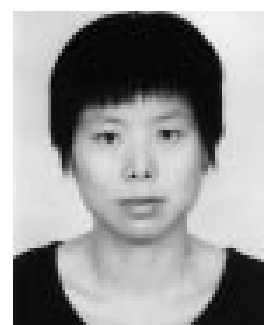

Xi Chen was born in Sichuan Province, China, in 1965. She received the B.Sc. and M.Eng. degrees in control theory and application from Nankai University, Tianjin, China, in 1986 and 1989, respectively.

From 1989 to 1996, she worked at the Software Engineering Institute at Beijing University of Aeronautics and Astronautics, Beijing, China. Currently, she is working toward the Ph.D. degree in the Department of Systems Engineering and Engineering Management, Chinese University of Hong Kong. Her research interests are in the areas of stochastic control, Petri nets, software engineering, and computer-aided design in control systems.

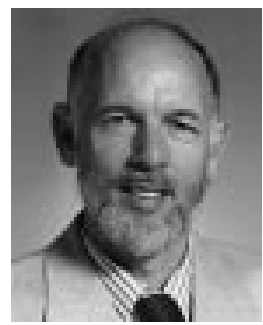

John B. Moore (S'66-M'68-SM'77-F'79) was born in China in 1941. He received the B.S., M.S., and $\mathrm{Ph} . \mathrm{D}$. degrees in electrical engineering from the University of Santa Clara, California, in 1963, 1964, and 1967 , respectively.

He was appointed Senior Lecturer at the Electrical Engineering Department, University of Newcastle in 1967, and promoted to Associate Professor in 1968, and Full Professor (Personal Chair) in 1973. From 1975-1979, he was Department Head. In 1982, he was appointed a Professorial Fellow in the Department of Systems Engineering, Research School of Physical Sciences, Australian National University, and promoted to Professor in 1990. He has been Head of the department since 1992, and is currently in the Research School of Information Sciences and Engineering. His current research is in control and communication systems, and signal processing. He is the coauthor of many books, including Linear Optimal Control (Englewood Cliffs, NJ: Prentice-Hall, 1971), Optimal Filtering (Englewood Cliffs, NJ: Prentice-Hall, 1979), Optimal Control-Linear Quadratic Methods (Englewood Cliffs, NJ: Prentice-Hall,1989), Optimization and Dynamical Systems (New York: Springer-Verlag, 1993), Hidden Markov Model Estimation and Control via Reference Methods (New York, SpringerVerlag, 1995), and High Performance Control (Boston, MA: Birkhäsuer 1997).

Dr. Moore is a Fellow of the Australian Academy of Technological Sciences and Engineering, and a Fellow of the Australian Academy of Science.

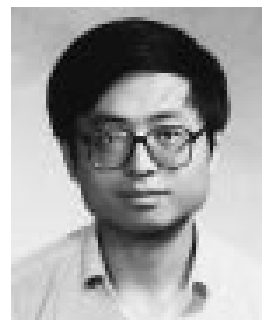

Xun Yu Zhou (M'97-SM'99) was born in Jiangsu Province, China in 1965. He received the Ph.D. degree in applied mathematics from Fudan University, Shangai, China, in 1989.

From 1989 to 1991 and from 1991 to 1993 , he did postdoctoral research at Kobe University, Kobe, Japan and the University of Toronto, Canada, respectively. He is currently an Associate Professor at the Chinese University of Hong Kong. A Principal Investigator of numerous grants, he has done extensive research in optimal stochastic controls, mathematical finance/insurance, and discrete-event manufacturing systems. He has published more than 40 papers in refereed journals, and his new book (coauthored with J. Yong) is entitled Stochastic Controls: Hamiltonian Systems and HJB Equations (New York: Springer-Verlag, 1999). He is on the editorial board of Operations Research.

Dr. Zhou was awarded the Alexander von Humboldt Fellowship of Germany in 1991 and is currently on the editorial board of the IEEE TRANSACTIONS ON AUTOMATIC CONTROL. 